$\mathrm{PSU} / \mathrm{TH} / 120$

\title{
Models for local ohmic quantum dissipation
}

\author{
Michael R. Gallis \\ Department of Physics, Pennsylvania State University, Schuylkill Campus, \\ Schuylkill Haven, Pennsylvania 17972 \\ Internet: MRG3@psuvm.psu.edu
}

\begin{abstract}
We propose a family of master equations for local quantum dissipation. The master equations are constructed in the form of Lindblad generators, with the constraints that the dissipation be strictly linear (i.e. ohmic), isotropic and translationally invariant. The resulting master equations are given in both the Schrödinger and Heisenberg forms. We obtain fluctuation-dissipation relations, and discuss the relaxation of average kinetic energy to effective thermal equilibrium values. We compare our results for one dimension to the Dekker master equation [H. Dekker, Phys. Rep. 80, 1 (1981)], which can be interpreted as a low length scale approximation of our model, as well as the Caldeira- Leggett master equation [A. O. Caldeira and A. J. Leggett, Physica (Utrecht) 121 A, 587 (1983)].
\end{abstract}

PACS number 3.65.-w, 3.65.Bz, 5.40.+j

Typeset Using REVTEX 


\section{INTRODUCTION}

Dissipative quantum systems and quantum brownian motion have long been of interest and a wide variety of techniques have been used to investigate them. [1,2], We will not attempt a comprehensive review, but extensive reviews can be found in Refs. [1] and [2]. For linear dissipation, one wishes to obtain dynamics for a quantum mechanical system which are in a sense generalizations of the classical equations of motion for linear dissipation:

$$
\begin{aligned}
m \dot{\mathbf{x}} & =\mathbf{p} \\
\dot{\mathbf{p}} & =-\frac{\eta}{m} \mathbf{p}+\mathbf{F} .
\end{aligned}
$$

The classical Langevin equations (and nonlinear generalizations) can be obtained by considering a particle interacting with an oscillator bath. [3] Quantum mechanical calculations

often begin with the same type of environment. For example, Caldeira and Leggett [4] use the influence functional technique of Feynman and Vernon [5] for a particle linearly coupled to a thermal bath of harmonic oscillators. The resulting (high temperature) master equation is given by

$\frac{\partial \rho}{\partial t}=\frac{1}{i \hbar}[H, \rho]+\frac{\eta}{i 2 m \hbar}[x,\{p, \rho\}]-\frac{\eta k_{B} T}{\hbar^{2}}[x,[x, \rho]]$.

An alternative approach by Dekker [1], uses the quantization of complex classical canonical coordinates and quantal noise operators, resulting in the master equation:

$$
\begin{aligned}
\frac{\partial \rho}{\partial t}= & \frac{1}{i \hbar}[H, \rho] \\
& -i \frac{\lambda}{\hbar}[x,\{p, \rho\}]+\frac{1}{\hbar^{2}}\left(D_{x p}+D_{p x}\right)[x,[p, \rho]] \\
& -\frac{D_{x x}}{\hbar^{2}}[p,[p, \rho]]-\frac{D_{p p}}{\hbar^{2}}[x,[x, \rho]] .
\end{aligned}
$$

Neither the Caldeira-Leggett nor the Dekker master equation is translationally invariant, and so cannot represent the effect on a quantum particle of a local interaction with a spatially homogenous environment. Caldeira and Leggett have pointed out that it is only strictly linear coupling (i.e. bilinear in system and oscillator bath coordinates) that can 
produce strictly linear dissipation. However, it may be true that other forms of coupling produce linear dissipation as an approximation which is valid over a wide range of conditions. Moreover, the dynamics of quantum coherence could be very different in a nonlinear (local coupling) versus a linear model [6.77]. It is the intent of this paper to show one method of constructing a master equation which reflects (in translational invariance) the symmetries of the environment and environment- system coupling which presumably give rise to the dissipative dynamics.

The approach to constructing the master equation is essentially algebraic. We begin with a master equation of the Lindblad form:

$$
\begin{aligned}
\frac{\partial \rho}{\partial t} & =L[\rho] \\
& =\frac{1}{i \hbar}[H, \rho]+\frac{1}{2 \hbar} \sum_{\mu}\left[V_{\mu} \rho, V_{\mu}^{\dagger}\right]+\left[V_{\mu}, \rho V_{\mu}^{\dagger}\right] \\
& =\frac{1}{i \hbar}[H, \rho]+\Delta L[\rho],
\end{aligned}
$$

which, for bounded $H,\left\{V_{\mu}\right\}$, generates a completely positive dynamical semigroup. [8] The generator for the corresponding Heisenberg picture is given by:

$$
\begin{aligned}
\frac{d O}{d t}-\frac{\partial O}{\partial t} & =L^{*}[O] \\
& =-\frac{1}{i \hbar}[H, O]+\frac{1}{2 \hbar} \sum_{\mu} V_{\mu}^{\dagger}\left[O, V_{\mu}\right]+\left[V_{\mu}^{\dagger}, O\right] V_{\mu} \\
& =-\frac{1}{i \hbar}[H, O]+\Delta L^{*}[O]
\end{aligned}
$$

Following an approach used by Sandulescu and Scutaru to generate the Dekker master equation, 9] we will operate under the assumption that this is also an appropriate form for a completely positive dynamical semigroup for unbounded operators. We will focus on the additional part of the master equation (Langevin equation) associated with dissipation, $\Delta L$ $\left(\Delta L^{*}\right)$

In Sec. [1] we will examine the classical Langevin equations for a particle coupled to a harmonic oscillator bath, which will help clarify the connection between first principle classical and quantum calculations, as well as the issues which arise for quantum generalizations 
of classical systems. In Sec. III, we will construct $\Delta L$ by choosing a particular form for the operators $\left\{V_{\mu}\right\}$, and establish some constraints on these operators from the requirement of linear dissipation. We will choose particular form for the operators which satisfy the constraints, yield a master equation which is translationally invariant, and which correspond to an isotropic environment. The results are presented in both the Schrödinger and Heisenberg forms. In Sec. IV we will derive and discuss fluctuation-dissipation relations for these models, as well as the time dependence of expectations of operators which are functions of position or momentum only. In Sec. V, we compare our results for one dimension to the Dekker and Caldeira- Leggett master equations. We will comment on our results in Sec. VI.

\section{CLASSICAL LANGEVIN EQUATIONS}

In this section, we review some aspects of Langevin equations which arise from interaction with a harmonic oscillator bath, essentially following Zwanzig [3]. The composite system has a lagrangian given by

$$
\begin{aligned}
L= & \frac{1}{2} m \dot{x}^{2}-U(\mathbf{x}) \\
& +\sum_{\mu} \frac{m_{\mu}}{2}\left[\dot{q}_{\mu}^{2}-\omega_{\mu}^{2}\left(q_{\mu}-a_{\mu}(\mathbf{x})\right)^{2}\right]
\end{aligned}
$$

where $\mathbf{x}$ is the position of the system of interest, and $q_{\mu}$ are the coordinates of the oscillators comprising the bath. The interaction terms are of the form $q_{\mu} m_{\mu} \omega_{\mu}^{2} a_{\mu}(\mathbf{x})$, and the additional

terms of the form $\frac{1}{2} m_{\mu} \omega_{\mu}^{2} a_{\mu}^{2}(\mathbf{x})$ are associated with regularization of the potential. The equation of motion for an individual oscillator is given by

$$
\ddot{q}_{\mu}=-\omega_{\mu}^{2} q_{\mu}+\omega_{\mu}^{2} a_{\mu}(\mathbf{x})
$$

which is simply a driven simple harmonic oscillator. The formal solution to the equation of motion is

$$
\begin{aligned}
q_{\mu}(t)= & q_{\mu}(0) \cos \omega_{\mu} t+\frac{\dot{q}_{\mu}(0)}{\omega_{\mu}} \sin \omega_{\mu} t \\
& +\int_{0}^{t} \sin \omega_{\mu}(t-s) \omega_{\mu} a_{\mu}(\mathbf{x}(s)) d s
\end{aligned}
$$


This becomes, after an integration by parts,

$$
\begin{aligned}
q_{\mu}(t)= & q_{\mu}(0) \cos \omega_{\mu} t+\frac{\dot{q}_{\mu}(0)}{\omega_{\mu}} \sin \omega_{\mu} t+a_{\mu}(x(t) \\
& -\cos \omega_{\mu}(t-s) a_{\mu}(x(0)) \\
& -\int_{0}^{t} \cos \omega_{\mu}(t-s) \frac{\partial a_{\mu}(\mathbf{x}(s))}{\partial x_{j}} \dot{x}_{j} d s
\end{aligned}
$$

The equation of motion for the particle is given (for the component $x_{i}$ ) by

$\ddot{x}_{i}(t)=-\frac{\partial U}{\partial x_{i}}+\sum_{\mu} m_{\mu} \omega_{\mu}^{2}\left(q_{\mu}-a_{\mu}(\mathbf{x})\right) \frac{\partial a_{\mu}(\mathbf{x})}{\partial x_{i}}$

Direct substitution of equation Eq. (2.4) into Eq. (2.5) yields an equation for motion for $\mathbf{x}$ which can be written

$$
\begin{aligned}
\ddot{x}_{i}(t)= & -\frac{\partial U(\mathbf{x})}{\partial x_{i}}+f_{i}(\mathbf{x}, t) \\
& -\int_{0}^{t} \eta_{i j}(\mathbf{x}(t), \mathbf{x}(s) ; t-s) \dot{x}_{j}(s) d s,
\end{aligned}
$$

(summation over $j$ implied) where $\mathbf{f}(\mathbf{x}, t)$ is interpreted as a fluctuating force given by

$$
\begin{aligned}
f_{i}(\mathbf{x}, t)=\sum_{\mu}\{ & m_{\mu} \omega_{\mu}^{2}\left[\left(q_{\mu}(0)-a_{\mu}(\mathbf{x}(0)) \cos \omega_{\mu} t\right.\right. \\
& \left.\left.+\frac{\dot{q}_{\mu}(0)}{\omega_{\mu}} \sin \omega_{\mu} t\right] \frac{\partial a_{\mu}(\mathbf{x}(t))}{\partial x_{i}}\right\}
\end{aligned}
$$

and the nonlinear dissipation kernel is given by

$$
\begin{array}{r}
\eta_{i j}(\mathbf{x}(t), \mathbf{x}(s) ; t-s)= \\
\sum_{\mu} m_{\mu} \omega_{\mu}^{2} \frac{\partial a_{\mu}(\mathbf{x}(t))}{\partial x_{i}} \frac{\partial a_{\mu}(\mathbf{x}(s))}{\partial x_{j}} \cos \omega_{\mu}(t-s) .
\end{array}
$$

The initial probability distribution of the oscillators determines the statistics of the fluctuating force. The oscillators are assumed to be independent and in a thermal state, with

$$
\left\langle q_{\mu}(0)-a_{\mu}(\mathbf{x}(0))\right\rangle=\left\langle\dot{q}_{\mu}(0)\right\rangle=0
$$

and 


$$
\begin{aligned}
\left\langle\frac{1}{2} m_{\mu} \omega_{\mu}^{2}\left(q_{\mu}(0)-a_{\mu}(\mathbf{x}(0))\right)^{2}\right\rangle & =\frac{1}{2 m_{\mu}}\left\langle\left(\dot{q}_{\mu}(0)\right)^{2}\right\rangle \\
& =\frac{1}{2} k_{B} T
\end{aligned}
$$

Under these conditions, we have the following fluctuation- dissipation relation:

$$
\begin{aligned}
\langle\mathbf{f}(\mathbf{x}, t)\rangle & =0 \\
\left\langle f_{i}(\mathbf{x}, t) f_{j}(\mathbf{y}, s)\right\rangle & =k_{B} T \eta_{i j}(\mathbf{x}, \mathbf{y} ; t-s) .
\end{aligned}
$$

With a typical Markov approximation, the width in time of $\eta_{i j}$ is taken to be small, effectively a delta function. The fluctuating force is effectively white noise, and the effective Langevin equation can be written:

$\ddot{x}_{i}(t)=-\frac{\partial U(\mathbf{x}(t))}{\partial x_{i}}+f_{i}(\mathbf{x}(t), t)-\bar{\eta}_{i j}(\mathbf{x}(t)) \dot{x}_{j}$

and the new fluctuation-dissipation relation is

$$
\left\langle f_{i}(\mathbf{x}, t) f_{j}(\mathbf{x}, s)\right\rangle=k_{B} T \bar{\eta}_{i j}(\mathbf{x}) \delta(t-s)
$$

The dependence of the dissipation on spatial correlations of the fluctuating forces has been lost. Since the particle can only be at one position at one time, these correlations play no role in the classical dynamics. Indeed, a wide variety of models can reduce to the same Langevin equation, if only in approximation. One could even assume a spatially homogeneous environment in which the $\bar{\eta}_{i j}$ is independent of $\mathbf{x}$, and the resulting Langevin equation to get linear dissipation. A quantum mechanical description of the particle and its dynamics includes superpostions between different positions, resulting in interference between different particle trajectories. One effect of the interaction with the oscillator bath is that the noise will result in a loss of quantum coherence. This loss of coherence will depend upon the spatial correlations of the noise [10,7]. In the the following sections we will explore master equations which have linear dissipation and include spatial correlation effects, and thus are candidate models for the effects on a quantum system resulting from local coupling to a homogeneous and isotropic environment. 


\section{CONSTRUCTION OF THE MASTER EQUATION}

To construct the master equation, we focus on the non unitary part of the evolution, that is,

$$
\Delta L^{*}[O] \equiv \frac{1}{2 \hbar} \sum_{\mu} V_{\mu}^{\dagger}\left[O, V_{\mu}\right]+\left[V_{\mu}^{\dagger}, O\right] V_{\mu}
$$

The time dependence of expectation values can immediately be obtained via

$\frac{d\langle O\rangle}{d t}=\left\langle\frac{\partial O}{\partial t}\right\rangle+\left\langle L^{*}[O]\right\rangle=\left\langle\frac{\partial O}{\partial t}\right\rangle+\left\langle\frac{i}{\hbar}[H, O]\right\rangle+\left\langle\Delta L^{*}[O]\right\rangle$

where $\langle O\rangle=\operatorname{Tr}[\rho O]$. We consider dissipation in the general case of $\mathrm{d}$ dimensions. For strictly linear dissipation, we require

$$
\begin{aligned}
& \Delta L^{*}[\mathbf{p}]=-\frac{\eta}{m} \mathbf{p}, \\
& \Delta L^{*}[\mathbf{x}]=0,
\end{aligned}
$$

which will produce quantum Langevin equations corresponding to Eq. (1.1). One could also consider more general forms for the linear dissipation where $\eta$ is replaced with a dissipation tensor, and the classical frictional force components would be given by $F_{i}=\eta_{i j} p_{j}$ (summation over $j$ ), corresponding to an environment which was anisotropic. The anisotropic case could be addressed by a simple generalization of what we present here.

The operators $\left\{V_{\mu}\right\}$ presumably could be expressed as functions of the position and momentum operators. This is the approach taken by Sandulescu and Scutaru citeScutaru, where $\left\{V_{\mu}\right\}$ were taken to be linear combinations of position and momentum. We will choose the particular form of $\left\{V_{\mu}\right\}$ to be at most linear in momentum, and write

$$
V_{\mu}=A_{\mu}(\mathbf{x})-\mathbf{B}_{\mu}(\mathbf{x}) \cdot \mathbf{p}
$$

were $\left\{A_{\mu}(\mathbf{x})\right\}$ and $\left\{\mathbf{B}_{\mu}(\mathbf{x})\right\}$ are as yet undetermined functions of $\mathbf{x}$. The choice of this form is practically dictated by the requirement that the dissipation be linear, as any higher powers of momentum would appear as nonlinear orders of momentum in the first part of Eq. 
(3.3). We can now use Eq. (3.1) and Eq. (3.3) to establish some conditions on the functions $\left\{A_{\mu}(\mathbf{x})\right\}$ and $\left\{B_{\mu i}(\mathbf{x})\right\}$. Evaluating Eq. (3.1) for a particular component of momentum, we take

$$
\Delta L^{*}\left[p_{n}\right]=\frac{1}{2 \hbar} \sum_{\mu} V_{\mu}\left[p_{n}, V_{\mu}^{\dagger}\right]+\left[V_{\mu}, p_{n}\right] V_{\mu}^{\dagger},
$$

substitute for $\left\{V_{\mu}\right\}$ with Eq. (3.4), and reorder the operators with all momentum terms to the right:

$$
\begin{aligned}
\Delta L^{*}\left[p_{n}\right]= & \frac{1}{2 i}\left(\sum_{\mu}\left(A_{\mu}^{\dagger} \frac{\partial A_{\mu}}{\partial x_{n}}-\frac{\partial A_{\mu}^{\dagger}}{\partial x_{n}} A_{\mu}\right)\right)-\frac{\hbar}{2} \sum_{\mu i}\left[\frac{\partial}{\partial x_{i}}\left(B_{\mu i}^{\dagger} \frac{\partial A_{\mu}}{\partial x_{n}}-\frac{\partial B_{\mu i}^{\dagger}}{\partial x_{n}} A_{\mu}\right)\right] \\
& -\frac{\hbar}{2} \sum_{\mu i j}\left\{\left[\frac{\partial}{\partial x_{i}}\left(B_{\mu i}^{\dagger} \frac{\partial B_{\mu j}}{\partial x_{n}}-\frac{\partial B_{\mu i}^{\dagger}}{\partial x_{n}} B_{\mu j}\right)\right] p_{j}\right\} \\
& -\frac{1}{2 i} \sum_{\mu i}\left[\left(B_{\mu i}^{\dagger} \frac{\partial A_{\mu}}{\partial x_{n}}-\frac{\partial B_{\mu i}^{\dagger}}{\partial x_{n}} A_{\mu}-B_{\mu i} \frac{\partial A_{\mu}^{\dagger}}{\partial x_{n}}+\frac{\partial B_{\mu i}}{\partial x_{n}} A_{\mu}^{\dagger}\right) p_{i}\right] \\
& +\frac{1}{2 i} \sum_{\mu i j}\left[\left(B_{\mu i}^{\dagger} \frac{\partial B_{\mu j}}{\partial x_{n}}-\frac{\partial B_{\mu i}^{\dagger}}{\partial x_{n}} B_{\mu j}\right) p_{i} p_{j}\right] .
\end{aligned}
$$

The resulting expression contains terms up to second order in momentum, and we equate each power to the corresponding term in Eq. (3.3), which produces, after some simplification, the following conditions on $\left\{A_{\mu}(\mathbf{x})\right\}$ and $\left\{B_{\mu i}(\mathbf{x})\right\}$ :

$$
\begin{aligned}
\sum_{\mu i j} B_{\mu i}^{*} \frac{\partial B_{\mu j}}{\partial x_{n}}-\frac{\partial B_{\mu i}^{*}}{\partial x_{n}} B_{\mu j} & =0 \\
\frac{1}{2 i} \sum_{\mu i}\left(B_{\mu i}^{*} \frac{\partial A_{\mu}}{\partial x_{n}}-\frac{\partial B_{\mu i}^{*}}{\partial x_{n}} A_{\mu}-B_{\mu i} \frac{\partial A_{\mu}^{*}}{\partial x_{n}}+\frac{\partial B_{\mu i}}{\partial x_{n}} A_{\mu}^{*}\right) & =\delta_{i n} \frac{\eta}{m} \\
\frac{1}{2 i} \sum_{\mu}\left(A_{\mu}^{*} \frac{\partial A_{\mu}}{\partial x_{n}}-\frac{\partial A_{\mu}^{*}}{\partial x_{n}} A_{\mu}\right)+\frac{\hbar}{2} \sum_{\mu i}\left[\frac{\partial}{\partial x_{i}}\left(B_{\mu i}^{*} \frac{\partial A_{\mu}}{\partial x_{n}}-\frac{\partial B_{\mu i}^{*}}{\partial x_{n}} A_{\mu}\right)\right] & =0 .
\end{aligned}
$$

To satisfy the second part of Eq. (3.3), we look at Eq. (3.1) for a particular component of the position operator to get

$$
\begin{aligned}
\Delta L^{*}\left[x_{n}\right]= & \frac{1}{2}\left\{\sum_{\mu j}\left[-\hbar \frac{\partial}{\partial x_{j}}\left(B_{\mu j}^{\dagger} B_{\mu n}\right)\right]\right. \\
& +\frac{1}{i} \sum_{\mu}\left[\left(A_{\mu}^{\dagger} B_{\mu n}-A_{\mu} B_{\mu n}^{\dagger}\right]\right. \\
& \left.\left.+\frac{1}{i} \sum_{\mu j}\left[B_{\mu n}^{\dagger} B_{\mu j}-B_{\mu j}^{\dagger} B_{\mu n}\right) p_{j}\right]\right\}
\end{aligned}
$$


The resulting constraint equations are

$$
\begin{aligned}
& \sum_{\mu j}\left[-\hbar \frac{\partial}{\partial x_{j}}\left(B_{\mu j}^{*} B_{\mu n}\right)\right]+ \frac{1}{i} \sum_{\mu}\left[\left(A_{\mu}^{*} B_{\mu n}-A_{\mu} B_{\mu n}^{*}\right]=0\right. \\
&\left.\sum_{\mu j}\left[B_{\mu n}^{*} B_{\mu j}-B_{\mu j}^{*} B_{\mu n}\right)\right]=0 .
\end{aligned}
$$

The conditions above by no means determine the functions $\left\{A_{\mu}(\mathbf{x})\right\}$ and $\left\{B_{\mu i}(\mathbf{x})\right\}$, but are constraints to be satisfied. The master equation we are generating is presumably a limiting form of the reduced dynamics of a composite quantum system, so we select a particular form of $\left\{A_{\mu}(\mathbf{x})\right\}$ and $\left\{B_{\mu i}(\mathbf{x})\right\}$ to reflect the form of the interaction between the system of interest and the environment degrees of freedom. For a local interaction with a field, the environment degrees of freedom are the particular modes of the field. A reasonable choice for these functions are then

$$
\begin{gathered}
\left\{A_{\mu}\right\}=\left\{a(\mathbf{k}) e^{i \mathbf{k} \cdot \mathbf{x}}\right\} \\
\left\{B_{\mu j}\right\}=\left\{b_{j}(\mathbf{k}) e^{i \mathbf{k} \cdot \mathbf{x}}\right\},
\end{gathered}
$$

where the index $\mu$ is replaced by a continuous $d$ dimensional wave vector $\mathbf{k}$, and sums over $\mu$ are replaced by integrations over $\mathbf{k}$. Some simplification occurs immediately since, with this choice, terms such as the product $A_{m}^{*} B_{m i}$ are independent of $\mathbf{x}$. The conditions expressed in Eq. (3.7) and Eq. (3.9) reduce to

$$
\begin{aligned}
\int d^{d} k b_{i}^{*}(\mathbf{k}) b_{j}(\mathbf{k}) k_{n} & =0 \\
\int d^{d} k 2 \operatorname{Re}\left(a^{*}(\mathbf{k}) b_{i}(\mathbf{k})\right) k_{n} & =\delta_{i n} \frac{\eta}{m} \\
\int d^{d} k\left|a^{*}(\mathbf{k})\right|^{2} k_{n} & =0 \\
\int d^{d} k \operatorname{Im}\left(a^{*}(\mathbf{k}) b_{i}(\mathbf{k})\right) & =0 \\
\int d^{d} k \operatorname{Im}\left(b_{j}^{*}(\mathbf{k}) b_{i}(\mathbf{k})\right) & =0 .
\end{aligned}
$$

To satisfy these conditions, we need $a(\mathbf{k})$ to be an even function of the components of $\mathbf{k}$, and $b_{i}(\mathbf{k})$ to be an odd function of $k_{i}$, but an even function of the other components of $\mathbf{k}$. To insure isotropy, we choose the form of the functions $a(\mathbf{k})$ and $a(\mathbf{k})$ as 


$$
\begin{gathered}
a(\mathbf{k})=\alpha(k) \\
b_{j}(\mathbf{k})=\beta(k) k_{j} .
\end{gathered}
$$

These functions can then be seen to satisfy the conditions (Eqs. (3.7) and (3.7)) by considering the symmetry of the even limits for the integrals over particular components of $\mathbf{k}$, along with the even or odd integrands. The dampening constant is given for this final choice by

$$
\begin{aligned}
\frac{\eta}{m} & =\int d^{d} k 2 \operatorname{Re}\left(\alpha^{*}(k) \beta(k)\right) k_{n}^{2} \\
& =\int d^{d} k 2 \operatorname{Re}\left(\alpha^{*}(k) \beta(k)\right) \frac{k^{2}}{d} .
\end{aligned}
$$

We can now write the nonunitary part of the evolution equations for the particular choice of $\left\{V_{\mu}\right\}$. For the Heisenberg picture (after some simplification using the symmetries of the functions $\alpha$ and $\beta$ ) we have for an arbitrary operator $O$,

$$
\begin{aligned}
\Delta L^{*}[O]= & \int d^{d} k \frac{|\alpha(k)|^{2}}{\hbar}\left(e^{-i \mathbf{k} \cdot \mathbf{x}} O e^{i \mathbf{k} \cdot \mathbf{x}}-O\right) \\
& +\int d^{d} k \frac{|\beta(k)|^{2}}{\hbar}\left(\mathbf{k} \cdot \mathbf{p} e^{-i \mathbf{k} \cdot \mathbf{x}} O e^{i \mathbf{k} \cdot \mathbf{x}} k \cdot \mathbf{p}-\frac{1}{2}\left\{O,(\mathbf{k} \cdot \mathbf{p})^{2}\right\}\right) \\
& -\int d^{d} k \frac{\operatorname{Re}\left(\alpha(k)^{*} \beta(k)\right)}{\hbar}\left(\left\{e^{-i \mathbf{k} \cdot \mathbf{x}} O e^{i \mathbf{k} \cdot \mathbf{x}}, \mathbf{k} \cdot \mathbf{p}\right\}\right) \\
& -\int d^{d} k \frac{i \operatorname{Im}\left(\alpha(k)^{*} \beta(k)\right)}{\hbar}\left(\left[e^{-i \mathbf{k} \cdot \mathbf{x}} O e^{i \mathbf{k} \cdot \mathbf{x}}, \mathbf{k} \cdot \mathbf{p}\right]\right),
\end{aligned}
$$

while for the Schrödinger picture we have

$$
\begin{aligned}
\Delta L[\rho]= & -\int d^{d} k \frac{|\alpha(k)|^{2}}{\hbar}\left(\rho-e^{i \mathbf{k} \cdot \mathbf{x}} \rho e^{-i \mathbf{k} \cdot \mathbf{x}}\right) \\
& -\int d^{d} k \frac{|\beta(k)|^{2}}{\hbar}\left(\frac{1}{2}\left\{(\mathbf{k} \cdot \mathbf{p})^{2}, \rho\right\}-\mathbf{k} \cdot \mathbf{p} e^{i \mathbf{k} \cdot \mathbf{x}} \rho e^{-i \mathbf{k} \cdot \mathbf{x}} \mathbf{k} \cdot \mathbf{p}\right) \\
& -\int d^{d} k \frac{\operatorname{Re}\left(\alpha(k)^{*} \beta(k)\right)}{\hbar}\left(\left\{\mathbf{k} \cdot \mathbf{p}, e^{i \mathbf{k} \cdot \mathbf{x}} \rho e^{-i \mathbf{k} \cdot \mathbf{x}}\right\}\right) \\
& -\int d^{d} k \frac{i \operatorname{Im}\left(\alpha(k)^{*} \beta(k)\right)}{\hbar}\left(\left[\mathbf{k} \cdot \mathbf{p}, e^{i \mathbf{k} \cdot \mathbf{x}} \rho e^{-i \mathbf{k} \cdot \mathbf{x}}\right]\right) .
\end{aligned}
$$

This is the main result of this paper: we have illustrated a method of constructing quantum master equations which are ohmic and translationally invariant. The translational invariance can easily be seen by replacing $\mathbf{x}$ with $\mathbf{x}^{\prime}+\mathbf{r}$, where $\mathbf{r}$ is a c-number ( $\mathbf{p}$ is unchanged by translation). The form of the nonunitary terms is unchanged. 


\section{DISSIPATION, FLUCTUATION AND EXPECTATIONS}

In this section, we will examine the implications of the dissipative part of the evolution given by Eq. (3.14) or Eq. (3.15). by making use of the Heisenberg evolution equations, where the time dependence of expectation values is given by:

$$
\frac{d\langle O\rangle}{d t}=\operatorname{Tr}\left[\rho\left(L^{*}[O]+\frac{\partial O}{\partial t}\right)\right] .
$$

Much immediate simplification can occur when one notes that $e^{i \mathbf{k} \cdot \mathbf{x}} O e^{-i \mathbf{k} \cdot \mathbf{x}}$ is simply a unitary transformation, corresponding to a translation of $\hbar \mathbf{k}$ in momentum space. Additional simplifications occur with the symmetries of the integrals over $\mathrm{k}$ and the particular form of $\alpha$ and $\beta$ as function of $k=|\mathbf{k}|$. From this, one can readily verify that $\Delta L^{*}(\mathbf{p})=-\frac{\eta}{m} \mathbf{p}$.

We now wish to look at the effect of the non unitary part of the evolution on kinetic energy. We find, with some simplification which arises from the isotropy of the model, that

$$
\begin{aligned}
\Delta L^{*}\left[\frac{P^{2}}{2 m}\right]= & \int d^{d} k \frac{|\alpha(k)|^{2}}{2 m} \hbar k^{2} \\
& +\left(\int d^{d} k \frac{|\beta(k)|^{2}}{d} \hbar k^{4}-2 \frac{\eta}{m}\right) \frac{P^{2}}{2 m} .
\end{aligned}
$$

This can be interpreted as a relaxation to thermal equilibrium, under certain circumstances. We can make the identifications

$$
\begin{aligned}
\gamma & \equiv 2 \frac{\eta}{m}-\int d^{d} k \frac{|\beta(k)|^{2}}{d} \hbar k^{4} \\
\frac{d}{2} k_{B} T & \equiv \frac{1}{\gamma} \int d^{d} k \frac{|\alpha(k)|^{2}}{2 m} \hbar k^{2},
\end{aligned}
$$

which determine an effective equilibrium temperature, provided $\gamma>0$. Since $\Delta L^{*}$ is linear and since $\Delta L^{*}(\mathrm{c}-$ number $)=0$, we can now rewrite Eq. (4.2) as

$$
\Delta L^{*}\left[\left(\frac{P^{2}}{2 m}-\frac{d}{2} k_{B} T\right)\right]=-\gamma\left(\frac{P^{2}}{2 m}-\frac{d}{2} k_{B} T\right) .
$$

The first term on the RHS of Eq. (3.15) can be identified as the effect of a random gaussian fluctuating potential. For a particle in a random potential with a translationally invariant two point correlation function with short relaxation time, approximated by 


$$
\langle V(\mathbf{x}, t) V(\mathbf{y}, s)\rangle=g(\mathbf{x}-\mathbf{y}) \delta(t-s)
$$

the master equation gains an additional term which is given in the position representation by [10,7]

$$
\langle\mathbf{x}|\Delta L(\rho)| \mathbf{y}\rangle=-\frac{1}{\hbar^{2}}(g(0)-g(\mathbf{x}-\mathbf{y})) \rho(\mathbf{x}, \mathbf{y})
$$

The position representation of the first term on the RHS of Eq. (3.15) is then given by

$$
\begin{aligned}
& -\left\langle\mathbf{x}\left|\int d^{d} k \frac{|\alpha(k)|^{2}}{\hbar}\left(\rho-e^{i \mathbf{k} \cdot \mathbf{x}} \rho e^{-i \mathbf{k} \cdot \mathbf{x}}\right)\right| \mathbf{y}\right\rangle \\
= & -\int d^{d} k \frac{|\alpha(k)|^{2}}{\hbar}\left(1-e^{i \mathbf{k} \cdot(\mathbf{x}-\mathbf{y})}\right) \rho(x, y),
\end{aligned}
$$

so that after some simplification, we can identify this as the effect of a random gaussian potential with correlation function given by

$$
\begin{array}{r}
\langle V(\mathbf{x}, t) V(\mathbf{y}, s)\rangle= \\
\hbar \int d^{d} k|\alpha(\mathbf{k})|^{2} \cos (\mathbf{k} \cdot(x-y)) \delta(t-s) .
\end{array}
$$

In terms of fluctuating forces, we can write

$$
\begin{aligned}
& \langle\mathbf{f}(\mathbf{x}, t) \cdot \mathbf{f}(\mathbf{y}, s)\rangle=\nabla_{x} \cdot \nabla_{y}\langle V(\mathbf{x}, t) V(\mathbf{y}, s)\rangle \\
& =\hbar \int d^{d} k|\alpha(k)|^{2} k^{2} \cos (\mathbf{k} \cdot(\mathbf{x}-\mathbf{y})) \delta(t-s) .
\end{aligned}
$$

The fluctuation-dissipation relation can now be written using the identifications made in Eq. (4.3),

$$
\begin{aligned}
\langle\mathbf{f}(\mathbf{x}, t) \cdot \mathbf{f}(\mathbf{x}, s)\rangle & =\langle\mathbf{f}(0, t) \cdot \mathbf{f}(0, s)\rangle \\
& =2 m \gamma \frac{d}{2} k_{B} T \delta(t-s) .
\end{aligned}
$$

We now wish to consider the expectations of more general quantities. For an arbitrary function of the position operator $g(\mathbf{x})$, it is straightforward to examine the time dependence of its expectation value via Eq. (4.1). Since $\mathrm{x}$ is unaffected by translations in momentum space, evaluating $\Delta L^{*}[g(\mathbf{x})]$ via Eq. (3.14) becomes 
$\Delta L^{*}[g(\mathbf{x})]=$

$$
\begin{aligned}
& +\int d^{d} k \frac{|\beta(k)|^{2}}{\hbar}\left(\mathbf{k} \cdot \mathbf{p} g(\mathbf{x}) \mathbf{k} \cdot \mathbf{p}-\frac{1}{2}\left\{g(\mathbf{x}),(\mathbf{k} \cdot \mathbf{p})^{2}\right\}\right) \\
& -\int d^{d} k \frac{\operatorname{Re}\left(\alpha(k)^{*} \beta(k)\right)}{\hbar}(\{g(\mathbf{x}), \mathbf{k} \cdot \mathbf{p}\}) \\
& -\int d^{d} k \frac{i \operatorname{Im}\left(\alpha(k)^{*} \beta(k)\right)}{\hbar}([g(\mathbf{x}), \mathbf{k} \cdot \mathbf{p}]),
\end{aligned}
$$

which is further simplified via the symmetries of the integrals over $\mathbf{k}$ to

$$
\begin{aligned}
\Delta L^{*}[g(\mathbf{x})] & =\int d^{d} k \frac{\hbar k^{2}|\beta(k)|^{2}}{2 d} \nabla^{2} g(\mathbf{x}) \\
& \equiv \frac{D}{2} \nabla^{2} g(\mathbf{x})
\end{aligned}
$$

effectively defining the diffusion constant $D$. The spreading of the density operator can then be characterized by

$$
\frac{d\left\langle\left(\mathbf{x}-\mathbf{x}_{o}\right)^{2}\right\rangle}{d t}=\left\langle\frac{i}{\hbar}\left[H,\left(\mathbf{x}-\mathbf{x}_{o}\right)^{2}\right]\right\rangle+D .
$$

For an arbitrary function of momentum, $h(\mathbf{p})$ we have

$$
e^{i \mathbf{k} \cdot \mathbf{x}} h(\mathbf{p}) e^{-i \mathbf{k} \cdot \mathbf{x}}=h(\mathbf{p}+\hbar \mathbf{k}),
$$

and $\Delta L^{*}[h(\mathbf{p})]$ becomes

$$
\begin{array}{r}
\Delta L^{*}[h(\mathbf{p})]= \\
\int d^{d} k \frac{|\alpha(k)|^{2}}{\hbar}(h(\mathbf{p}+\hbar \mathbf{k})-h(\mathbf{p})) \\
+\int d^{d} k \frac{|\beta(k)|^{2}}{\hbar}\left[(\mathbf{k} \cdot \mathbf{p})^{2}(h(\mathbf{p}+\hbar \mathbf{k})-h(\mathbf{p}))\right] \\
-\int d^{d} k \frac{2 \operatorname{Re}\left(\alpha(k)^{*} \beta(k)\right)}{\hbar}(\mathbf{k} \cdot \mathbf{p}) h(\mathbf{p}+\hbar \mathbf{k}) .
\end{array}
$$

\section{COMPARISON WITH DEKKER AND}

\section{CALDEIRA-LEGGETT MASTER EQUATIONS}

It is useful to take a look at Sandulescu and Scutaru's construction of the Dekker master equation in order to make comparisons with our results. In section two, we were largely 
followed their construction. Their choice for the operators $\left\{V_{\mu}\right\}$ were linear combinations of position and momentum operators,

$$
V_{i}=a_{i} x+b_{i} p
$$

while in our d-dimensional generalization, we considered operators of the form:

$$
V_{\mathbf{k}}=\alpha(\mathbf{k}) e^{i \mathbf{k} \cdot \mathbf{x}}-\beta(\mathbf{k}) e^{i \mathbf{k} \cdot \mathbf{x}} \mathbf{k} \cdot \mathbf{p}
$$

It is possible to consider the Dekker equation as a low length scale limit of our result. One can imagine that this will correspond to a system which is well localized, perhaps by the potential of the system of interest. We can take the origin to be centered in the region of localization, noting that translations will not affect the form of $\Delta L$. The length scale is determined the physical extent of this region of localization. A short length scale approximation can be considered as a long wavelength (for the environment modes) approximation via the "typical" wave numbers in Eq. (5.2). To make this approximation we expand in powers of $k$. To second order, we have

$$
e^{i \mathbf{k} \cdot \mathbf{x}} O e^{-i \mathbf{k} \cdot \mathbf{x}} \cong O+i[\mathbf{k} \cdot \mathbf{x}, O]-\frac{1}{2}[\mathbf{k} \cdot \mathbf{x},[\mathbf{k} \cdot \mathbf{x}, O]]
$$

With simplifications (due to the symmetry in our choice of $\alpha$ and $\beta$ ), $\Delta L$ becomes

$$
\begin{aligned}
\Delta L[\rho] \cong & -\int d^{d} k \frac{|\alpha(k)|^{2}}{2 \hbar}[\mathbf{k} \cdot \mathbf{x},[\mathbf{k} \cdot \mathbf{x}, \rho]] \\
& -\int d^{d} k \frac{|\beta(k)|^{2}}{2 \hbar}[\mathbf{k} \cdot \mathbf{p},[\mathbf{k} \cdot \mathbf{p}, \rho]] \\
& -\int d^{d} k \frac{i \operatorname{Re}\left(\alpha(k)^{*} \beta(k)\right)}{\hbar}[\mathbf{k} \cdot \mathbf{x},\{\mathbf{k} \cdot \mathbf{p}, \rho\}] \\
& +\int d^{d} k \frac{\operatorname{Im}\left(\alpha(k)^{*} \beta(k)\right)}{\hbar}[\mathbf{k} \cdot \mathbf{x},[\mathbf{k} \cdot \mathbf{p}, \rho]] .
\end{aligned}
$$

In 1 dimension, this corresponds to the Dekker master equation, with

$$
\begin{aligned}
\Delta L[\rho]= & -i \frac{\lambda}{\hbar}[x,\{p, \rho\}]+\frac{1}{\hbar^{2}}\left(D_{x p}+D_{p x}\right)[x,[p, \rho]] \\
& -\frac{D_{x x}}{\hbar^{2}}[p,[p, \rho]]-\frac{D_{p p}}{\hbar^{2}}[x,[x, \rho]],
\end{aligned}
$$


where the parameters are given by

$$
\begin{aligned}
D_{p p} & =\int d^{d} k \frac{\hbar|\alpha(k)|^{2}}{2} k^{2} \\
D_{x x} & =\int d k \frac{\hbar|\beta(k)|^{2}}{2} k^{2} \\
\lambda & =\int d k i \operatorname{Re}\left(\alpha(k)^{*} \beta(k)\right) k^{2}=\frac{\eta}{2 m} \\
\left(D_{x p}+D_{p x}\right) & =\int d k \hbar \operatorname{Im}\left(\alpha(k)^{*} \beta(k)\right) k^{2} .
\end{aligned}
$$

For the Caldeira-Leggett master equation, we have

$$
\Delta L[\rho]=\frac{\eta}{i 2 m \hbar}[x,\{p, \rho\}]-\frac{\eta k_{B} T}{\hbar^{2}}[x,[x, \rho]],
$$

which can be viewed as a low momentum approximation (ignoring second order momentum terms in $\Delta L$ ) of the Dekker master equation with $D_{x p}=D_{p x}=0$. If we make similar approximations for our master equation, without making short length scale approximations, we have

$$
\begin{array}{r}
\Delta L[\rho]=-\int d^{d} k \frac{|\alpha(k)|^{2}}{\hbar}\left(\rho-e^{i \mathbf{k} \cdot \mathbf{x}} \rho e^{-i \mathbf{k} \cdot \mathbf{x}}\right) \\
-\int d^{d} k \frac{\operatorname{Re}\left(\alpha(k)^{*} \beta(k)\right)}{\hbar}\left(\left\{\mathbf{k} \cdot \mathbf{p}, e^{i \mathbf{k} \cdot \mathbf{x}} \rho e^{-i \mathbf{k} \cdot \mathbf{x}}\right\}\right) .
\end{array}
$$

For this case, the fluctuation-dissipation relations are of a more familiar form, relating the effective temperature to the correlation of the fluctuating potentials, where the equivalent relations to Eqs. (4.3) and(4.10) become

$$
\begin{aligned}
\gamma & =2 \frac{\eta}{m} \\
\frac{d}{2} k_{B} T & \equiv \frac{1}{\gamma} \int d^{d} k \frac{|\alpha(k)|^{2}}{2 m} \hbar k^{2},
\end{aligned}
$$

and

$$
\begin{aligned}
\langle\mathbf{f}(x, t) \cdot \mathbf{f}(x, s)\rangle & =\langle\mathbf{f}(0, t) \cdot \mathbf{f}(0, s)\rangle \\
& =2 m \gamma \frac{d}{2} k_{B} T \delta(t-s) \\
& =4 \eta \frac{d}{2} k_{B} T \delta(t-s) .
\end{aligned}
$$




\section{DISCUSSION}

We have introduced a method of construction of models for linear (i.e. ohmic) dissipation for a quantum system for arbitrary dimension. The model is completely specified by identifying the complex functions $a(\mathbf{k})$ and $\mathbf{b}(\mathbf{k})$. Although we have focused on isotropic dissipation, anisotropic dissipation can readily be introduced by retaining the direction dependence of $a$ and $b$ on $\mathbf{k}$. We have identified the phenomenological dissipation constant, and effective temperature, as well as the spatial correlation function for the fluctuating forces. Generalizations of this approach might also include considering other sets of non-trivial functions for $\left\{A_{\mu}(\mathbf{x})\right\}$ and $\left\{B_{\mu i}(\mathbf{x})\right\}$, so that the conditions expressed in Eqs. (3.7) and (3.9) are satisfied when averaged over some characteristic length scale.

The models we have introduced are natural generalizations of the Dekker master equation, and in one dimension, reduce to it in a short length scale approximation. Conceivable, one could construct a more general master equation using Dekker's noise operators by including spatial correlation effects. The Caldeira- Leggett master equation can be viewed as a short-length scale, small momentum approximation to our result.

Caldeira and Leggett have pointed out that the only coupling to an oscillator bath which exactly produces ohmic dissipation is linear coupling (in both system and environment coordinates). However, one might expect that nonlinear coupling may provide dissipation which is linear under a wide range of approximations. The models we have proposed here are intended to anticipate the form of the dynamics which would result from local coupling to an environment. We have previously argued that the effect on the dynamics of quantum coherence may differ considerably from that which can be obtained by linear coupling. [6,7]

\section{ACKNOWLEDGMENTS}

I would like to acknowldge the hospitality of the Aspen Center for Physics, where some of where part of this work was done. I am also grateful to Salman Habib for several enlightening 
conversations. 


\section{REFERENCES}

[1] H Dekker, Phys. Rep. 80, 1 (1981).

[2] H. Grabert, et. al. Phys. Rep. 168,115 (1988).

[3] R. Zwanzig, J. Stat. Phys 9, 215 (1973).

[4] A. O. Caldeira and A. J. Leggett, Physica (Utrecht) 121 A, 587 (1983).

[5] R. P. Feynman and F. L. Vernon Jr., Ann. Phys. (NY) 24, 118 (1963).

[6] M. R. Gallis and G. N. Fleming, Phys. Rev. A 42, 38 (1990).

[7] M. R. Gallis, Phys. Rev. A 45,47 (1992).

[8] G. Lindblad, Commun. Math. Phys. 48, 119 (1976).

[9] A. Sandulescu and H. Scutaru, Ann. Phys. (NY) 173, 277 (1987).

[10] L. Diosi, Phys. Lett. A 112, 288 (1985). 